\title{
Striatal amyloid is associated with tauopathy and memory decline in familial Alzheimer's disease
}

Bernard J. Hanseeuw ${ }^{1,2}$, Francisco Lopera ${ }^{3}$, Reisa A. Sperling ${ }^{1,4}$, Daniel J. Norton ${ }^{1}$, Edmarie Guzman-Velez ${ }^{1}$, Ana Baena', Enmanuelle Pardilla-Delgado ${ }^{1}$, Aaron P. Schultz , Jennifer Gatchel', David Jin ${ }^{1}$, Kewei Chen ${ }^{5}$, Eric M. Reiman ${ }^{5}$, Keith A. Johnson ${ }^{1,4+}$ and Yakeel T. Quiroz ${ }^{1 *+}$

\begin{abstract}
Background: Autosomal dominant Alzheimer's disease (ADAD) is distinguished from late-onset AD by early striatal amyloid- $\beta$ deposition. To determine whether striatal Pittsburgh compound B (PiB)-PET measurements of amyloid- $\beta$ can help predict disease severity in ADAD, we compared relationships of striatal and neocortical PiB-PET to age, tau-PET, and memory performance in the Colombian Presenilin 1 E280A kindred.

Methods: Fourteen carriers (age $=28-42$, Mini-Mental State Examination $=26-30$ ) and 20 age-matched noncarriers were evaluated using PiB, flortaucipir (FTP; tau), and memory testing (CERAD Word List Learning). PiB-PET signal was measured in neocortical and striatal aggregates. FTP-PET signal was measured in entorhinal cortex.

Results: Compared to non-carriers, mutation carriers had age-related elevations in both neocortical and striatal PiB binding. The PiB elevation in carriers was significantly greater in the striatum than in the neocortex. In mutation carriers, PiB binding in both the neocortex and the striatum is related to entorhinal FTP; however, the association was stronger with the striatum. Only striatal PiB was associated with worse memory. Remarkably, PiB binding in the striatum, but not in the neocortex, predicted entorhinal FTP and lower memory scores after adjusting for age, indicating that striatal PiB identified the carriers with the most severe disease.
\end{abstract}

Conclusions: Based on these preliminary cross-sectional findings, striatal PiB-PET measurements may offer particular value in the detection and tracking of preclinical ADAD, informing a mutation carrier's prognosis and evaluating amyloid- $\beta$-modifying ADAD treatments.

Keywords: Early-onset autosomal dominant Alzheimer's disease, Striatum, Tau PET, Amyloid PET, Memory

\section{Background}

Presenilin 1 (PSEN1), PSEN2, and amyloid precursor protein (APP) mutations have been shown to cause autosomal dominant Alzheimer's disease (ADAD). Because the age of dementia onset is highly predictable in ADAD, studying cognitively unimpaired mutation carriers allows to characterize the temporal sequence of ADAD biomarker changes prior to cognitive decline and to inform the design of ADAD prevention trials.

\footnotetext{
* Correspondence: yquiroz@mgh.harvard.edu

${ }^{\dagger}$ Keith A. Johnson and Yakeel T. Quiroz contributed equally to this work.

${ }^{1}$ Massachusetts General Hospital, Harvard Medical School, 149 13th Street,

Suite 10.014, Charlestown, Boston, MA 02119, USA

Full list of author information is available at the end of the article
}

Recent research in families with ADAD supports the hypothesis that amyloid- $\beta$ (A $\beta$ ) plaques accumulate early in the disease process and are followed by extensive tauopathy, neurodegeneration, and progressive cognitive decline [1-4]. Amyloid Pittsburgh compound $\mathrm{B}$ (PiB)-PET [5-7] and autopsy [5] data have raised the possibility that some forms of ADAD may be associated with greater fibrillar $A \beta$ deposition in the striatum than the neocortex in the preclinical stages of ADAD, a pattern that is not typically observed in sporadic AD [8]. Because of this regional pattern, we examined whether a striatal PiB biomarker had different predictive value for disease severity, as assessed by tau-PET and memory performance, compared to the most commonly

(c) The Author(s). 2019 Open Access This article is distributed under the terms of the Creative Commons Attribution 4.0 International License (http://creativecommons.org/licenses/by/4.0/), which permits unrestricted use, distribution, and 
used neocortical PiB biomarker, in cognitively unimpaired PSEN1-E280A mutation carriers and non-carriers from the largest known ADAD kindred due to a single-mutation.

\section{Methods \\ Participants}

Data from 34 cognitively unimpaired individuals, living in the metropolitan area of the Aburra Valley in Colombia and descending from a common ancestor, were analyzed. There were 14 carriers of the PSEN1-E280A mutation and 20 non-carriers. The median age of the carriers was 35 years old [28-42]. Carriers in this kindred develop mild cognitive impairment at a mean age of 45 [9] (SD 5.3, range 32-62) [10]. All participants scored a Mini-Mental State Examination (MMSE) of 26 or above and a global Clinical Dementia Rating (CDR) of 0. Twenty-one participants were previously described [3].

\section{Procedures}

Cognitive measures were undertaken at the University of Antioquia (Colombia) and included the MMSE, the $\mathrm{CDR}$, and the word list learning delayed recall memory score from the Consortium to Establish a Registry for Alzheimer Disease (CERAD), validated in a Colombian population [11]. Within 2 months of testing, participants traveled to the Massachusetts General Hospital (MGH) in Boston for brain imaging. MRI was acquired using a Siemens Tim Trio 3-T and PET using a Siemens HR+. Forty- to 60-min dynamic [11C]-Pittsburgh Compound $B$ (PiB; fibrillar $A \beta$ ) and 80-100-min [18F]-flortaucipir (FTP; tau) PET scans were acquired, and regional-to-cerebellar gray matter PiB distribution volume ratios (DVRs) and FTP standard uptake volume ratios (SUVRs) were computed as previously described [3]. Freesurfer v.5, co-registered T1-weighted MRIs, and automated regions-of-interest were used to mean neocortical and striatal PiB-DVRs, with no partial volume correction. The neocortical DVRs were generated from bilateral frontal, lateral temporal, and retrosplenial cortices; striatal DVRs were computed from bilateral caudate and putamen. FTP-SUVRs were characterized in the entorhinal cortex ROI, since this region was found to be associated with early FTP-SUVR increases over age in our PSEN1-E280A kindred [3].

Participants provided written informed consent, and approvals were obtained from the University of Antioquia Ethics Committee and the MGH Institutional Review Board. Participants and clinical investigators were blinded to the participants' mutation status.

\section{Statistics}

$T$ tests and $X^{2}$ tests were used to compare the data of mutation carriers and non-carriers. ANOVAs with repeated measures compared PiB-DVRs in the neocortex and striatum in the mutation carriers and non-carriers. Regions-of-interest (neocortex and striatum) were used as repeated factors. Linear regressions estimated the rate of age-related increase in PiB and FTP-PET uptake, and the rate of age-related memory decline. Age-by-group interactions determined the age of PET signal detection, and a 5000-iterations bootstrapping procedure estimated 95th percentile confidence intervals around this age. Spearman's $R^{2}$ were used to characterize the extent to which neocortical and striatal PiB-DVRs were related to entorhinal cortex FTP-SUVRs and CERAD memory scores.

\section{Results}

\section{Groups' comparisons}

Demographic characteristics, MMSE scores, and CERAD memory scores were not significantly different between mutation carriers and non-carriers. In contrast, entorhinal FTP was higher in the 14 carriers (Table 1), indicating that they had early tau pathology in the absence of detectable cognitive decline.

Neocortical and striatal PiB-DVRs were higher in mutation carriers than in non-carriers $(p<0.0001)$. Importantly, the between-group difference in $\mathrm{PiB}$ uptake (striatum: 0.43DVR between carriers and non-carriers, neocortex: $0.25 \mathrm{DVR}$ ) was greater in the striatum than in

Table 1 Participant characteristics

\begin{tabular}{llll}
\hline $\begin{array}{l}\text { Mean value } \\
\text { (SD) }\end{array}$ & Carriers & $\begin{array}{l}\text { Non-carriers } \\
N=14\end{array}$ & Statistics \\
\hline Age & 35.0 & 35.8 & $t=-0.5$ \\
Years & $(5.1)$ & $(5.0)$ & $p=0.65$ \\
Education & 11.1 & 10.5 & $t=+0.4$ \\
Years & $(4.1)$ & $(4.0)$ & $p=0.69$ \\
Female & 9 & 10 & $x^{2}=0.7$ \\
\% & $(64.3 \%)$ & $(50.0 \%)$ & $p=0.41$ \\
MMSE & 28.5 & 28.9 & $t=-1.0$ \\
& $(1.1)$ & $(0.9)$ & $p=0.34$ \\
CERAD & 6.6 & 7.5 & $t=-1.4$ \\
Recall & $(2.3)$ & $(1.2)$ & $p=0.17$ \\
Entorhinal & 1.24 & 0.99 & $\boldsymbol{t}=+\mathbf{3 . 9}$ \\
FTP-SUVr & $(0.27)$ & $(0.08)$ & $\boldsymbol{p}=\mathbf{0 . 0 0 0 5}$ \\
Neocortical & 1.27 & 1.02 & $\boldsymbol{t}=+\mathbf{7 . 1}$ \\
PiB-DVR & $(0.15)$ & $(0.02)$ & $\boldsymbol{p}<\mathbf{0 . 0 0 0 1}$ \\
Striatal & 1.67 & 1.24 & $\boldsymbol{t}=+\mathbf{7 . 7}$ \\
PiB-DVR & $(0.25)$ & $(0.05)$ & $\boldsymbol{p}<\mathbf{0 . 0 0 0 1}$ \\
\hline
\end{tabular}

Significant $p$-values are correctly highlighted in bold 
the neocortex (region-by-group interaction: $\mathrm{F}=17.1$, $p=0.0002$ ). Note that the non-carriers also had higher striatal than neocortical uptake (repeated-measure ANOVA: $\mathrm{F}=556.4, p<0.0001$ ), presumably reflecting striatal non-specific binding to white matter.

\section{Relationships to age}

In the carriers, neocortical and striatal PiB-DVRs, entorhinal FTP-SUVRs, and memory scores were age-related. In the non-carriers, no age relationships were observed (all $p$ 's $>0.20$ ). All the age-by-group interactions were significant, such that carriers had greater associations between age and PiB-DVRs (both $p<0.0001$ ), entorhinal FTP-SUVRs $(p=0.008)$, and memory scores $(p=0.009)$, than non-carriers. The age-by-group interactions demonstrated that neocortical and striatal PiB-DVRs were higher from age 28 [CI95 27-30], entorhinal FTP-SUVRs were higher from age 32 [30-33], and memory scores were lower in carriers older than 37 [[CI95 34-40] (Fig. 1).

Although $\mathrm{PiB}$ increased more with age in the striatum $(+0.040 \mathrm{DVR} /$ year $)$ than in the neocortex $(+0.026 \mathrm{DVR} /$ year), the age relationships were not statistically different between regions (age-by-region interaction in the carriers only: $\mathrm{F}=2.0, \quad p=0.18 ;$ age-by-region-by-group interaction: $\mathrm{F}=2.2, p=0.15$ ).

\section{Relationships to tauopathy and memory}

In the carriers, neocortical PiB-DVRs were correlated with entorhinal FTP-SUVRs, but not significantly with lower memory scores (Fig. 2-left, $p=0.14$ ). In contrast, striatal PiB-DVRs were significantly correlated with both entorhinal FTP-SUVRs and lower memory scores. Striatal PiB-DVRs were distinguished from neocortical PiB-DVRs by significantly greater associations with entorhinal cortex FTP-SUVRs (ANOVA: $p=0.02$ ) and lower memory scores (Fig. 2-right, $p=0.02$ ). In the non-carriers, neocortical and striatal PiB-DVRs were not significantly correlated with FTP-SUVRs or memory scores (all $R^{2}<0.01, p>0.7$ ). The preferential (striatal $>$ neocortical) association between PiB-DVRs and lower memory scores was significantly greater in the carriers than in the non-carriers $(\mathrm{F}=5.4, p=0.03)$.

To test whether higher striatal PiB-DVRs were associated with tauopathy and memory in carriers of the same age, we adjusted the previously observed associations for age and demonstrated that higher striatal PiB-DVRs were associated with higher entorhinal FTP-SUVRs (age-adjusted partial $R^{2}=0.50, p=0.007$ ), and lower memory scores $\left(R^{2}=0.28, p=0.09\right.$, trend-level), independently of age.

Given the possible confounds of using the cerebellum as reference region, we also compared neocortical and striatal PiB using pons as a reference. Results with pons were very similar to the ones with the cerebellum (e.g., the statistical associations between striatal $\mathrm{PiB}$ and entorhinal FTP/memory performance that we observed with cerebellum as reference region were also observed with pons as reference).
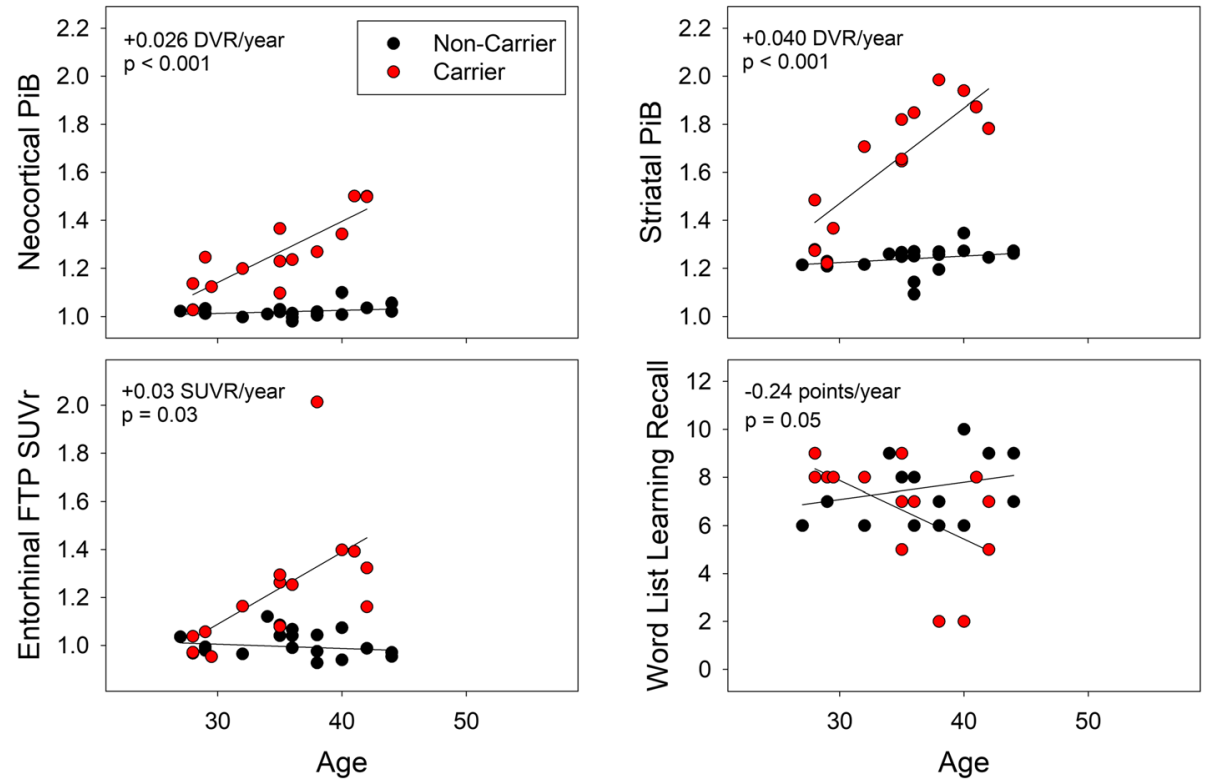

Fig. 1 Neocortical and striatal PiB (amyloid), entorhinal FTP (tau), and memory scores as a function of age. Linear regressions estimated the rate of age-related increase in PiB and FTP-PET uptake, and the rate of age-related memory decline in mutation carriers (red) and non-carriers (black). All age-related rates were significantly greater in carriers than non-carriers. The age-related rate in carriers is provided in red font 



Fig. 2 Associations between regional PiB measures, entorhinal FTP, and episodic memory. Spearman's $R^{2}$ were computed between PiB uptake and FTP uptake in the entorhinal cortex (top row) or memory scores (bottom row) in the mutation carriers. In the carriers, significant correlations were observed for all correlations other than the correlation between neocortical PiB-DVR and lower recall memory $(p=0.14)$. There were no significant correlations in the non-carriers. The preferential (striatal>neocortical) association between PiB-DVRs is illustrated on the right. The Fstatistics provided is an ANOVA with repeated measures comparing the association of both PiB regions with the outcomes, in the carriers only

\section{Discussion}

In this cross-sectional study, we characterized and compared the extent to which striatal and neocortical PiB-PET measurements of fibrillar $A \beta$ were associated with age, entorhinal tau-PET, and memory scores in cognitively unimpaired Colombian PSEN1-E280A mutation carriers and non-carriers. While striatal and neocortical $\mathrm{PiB}$ were both significantly associated with age in the mutation carrier group, the striatal measure was distinguished by significantly greater associations with tau burden and memory scores than the neocortical measure. This study supports the value of striatal PiB-PET measurements to predict disease severity in the preclinical stage of ADAD.

The association of striatal PiB with entorhinal FTP and memory scores survived age-adjustment, indicating that striatal $A \beta$ deposition could potentially be used to predict short-term clinical progression in carriers of the same age. Longitudinal and treatment studies are ongoing to compare striatal and neocortical $\mathrm{PiB}$ measurements in their ability to track $\mathrm{A} \beta$ plaque deposition, evaluate $A \beta$ treatments, and provide prognostic information in cognitively unimpaired ADAD mutation carriers.

Additional studies are needed to clarify the extent to which these findings are relevant to other A $\beta$-PET tracers, which may be less sensitive to the detection of preclinical and particularly diffuse $A \beta$ plaques.
Unlike [11C]-PiB observations in other ADAD families [5-7], a previous study of the Colombian kindred observed similar age-related $\mathrm{A} \beta$ deposition in the cortex and striatum, using [18F]-florbetapir [2]. The mechanisms leading to early elevations in striatal PiB-PET in ADAD are not fully elucidated, but higher affinity of $[11 \mathrm{C}]-\mathrm{PiB}$ for diffuse plaques, predominantly observed in the striatum, as opposed to neuritic plaques that are more often seen in the neocortex [5], was suggested as a potential mechanism for the discrepant results obtained with the different tracers. Higher striatal non-specific binding with florbetapir might also account for a lower sensitivity [8]. However, case reports [12] indicate that [18F]-compounds are able to visualize early striatal $A \beta$ in ADAD. Using $\mathrm{PiB}$, we observed higher $\mathrm{A} \beta$ burden in the striatum than in the neocortex, and the rate of age-related $A \beta$ deposition was nominally greater in the striatum, although not significantly greater, than in the neocortex. In the Colombian kindred, $A \beta$ deposits thus in both regions, with a marginally faster rate in the striatum. Besides the tracers used, ADAD studies also differ in the mutations responsible for the disease [6], and regional $A \beta$ deposition might be dependent on the specific mutation type. A previous study including carriers of different PSEN1 mutations indeed observed regional heterogeneity [13]. Further research, including studies from the Dominantly Inherited Alzheimer's Network (DIAN), could help 
clarify the extent to which findings in PSEN1-E280A mutation carriers are relevant to those with other mutations. Recent data suggest that, on average, individuals included in the DIAN study demonstrate amyloid accumulation in precuneus before the striatum [14]. However, the relations of amyloid in both regions with tauopathy and memory performance still require evaluation.

\section{Conclusions}

In this cross-sectional study of the Colombian kindred, striatal PiB-PET measurements better correlated with tau-PET measurements and memory performances than neocortical PiB-PET measurements. Future studies will evaluate the value of striatal PiB-PET to track $A \beta$ accumulation in longitudinal preclinical ADAD studies, to inform a mutation carrier's prognosis, and to evaluate $\mathrm{A} \beta$-modifying ADAD treatments.

\section{Consent}

Written informed consents were obtained from all the participants for the publication of this manuscript. Copies of the written consents are available for review by the Editor-in-Chief of this journal. Approvals were obtained from the University of Antioquia Ethics Committee and the MGH Institutional Review Board before that participants underwent any procedures.

\section{Abbreviations}

ADAD: Autosomal dominant Alzheimer's disease; APP: Amyloid precursor protein; $A \beta$ : Amyloid- $\beta$; CDR: Clinical Dementia Rating; CERAD: Consortium to establish a registry for Alzheimer's disease; DVR: Distribution volume ratio; FTP: Flortaucipir; MGH: Massachusetts General Hospital; MMSE: Mini-Mental State Examination; PiB: Pittsburgh Compound B; PSEN: Presenilin;

SUVr: Standard uptake volume ratio

\section{Acknowledgements}

The authors thank the PSEN1 Colombian families for contributing their valuable time and effort, without which this study would not have been possible. We thank the research assistants Alex Navarro, Francisco Piedrahita, Liliana Lopez and Lina Velilla from the Group of Neuroscience of Antioquia for their help coordinating study visits in Colombia. We thank the research assistants Justin Sanchez, Fred Uquillas, Olivia Hampton, Jairo Martinez and Valeria Torres from Massachusetts General Hospital for their help coordinating visits and neuroimaging examinations in Boston.

\section{Funding}

This study was supported by grants from the $\mathrm{NIH}$ Office of the Director (DP5OD019833 [YTQ]), the National Institute on Aging (R01 AG054671 [YTQ], P30 AG19610 [EMR] and R01AG055444 [EMR]), the Massachusetts General Hospital ECOR (1200-228010 [YTQ] and 1200-228767 [YTQ]), the state of Arizona (EMR), Banner Alzheimer's Foundation, NOMIS Foundation, Flinn Foundation, and FIL Foundation. Dr. Reiman is a principal investigator of the Alzheimer's Prevention Initiative (API) Autosomal Dominant AD Trial, which is supported by NIA, philanthropy, Genentech, and Roche. He is a scientific advisor to Roche, which covers travel expenses only. His organizations have contracts with Lilly/Avid for the performance of florbetapir and flortaucipir PET scans. Dr. Hanseeuw is supported by the Belgian Fund for Scientific Research (FNRS grant \#SPD28094292) and the Belgian Foundation for Alzheimer Research (SAO-FRA: grant P16008). The authors do not report conflicts of interest.

\section{Availability of data and materials}

The dataset used in the current study are available from the corresponding author on reasonable request, as long as data transfer is in agreement with USA legislation on data protection regulation.

\section{Authors' contributions}

BJH designed the study, analyzed the data, conducted the statistical analyses, and drafted the manuscript. FL designed the study, acquired the cognitive data, and revised the manuscript. RAS designed the study, acquired the PET data, and revised the manuscript. DN acquired the data, made the figures, and revised the manuscript. EGV acquired the PET data and revised the manuscript. $A B$ and EPD acquired the PET data and revised the manuscript. APS processed the PET data and revised the manuscript. JG revised the manuscript and provided intellectual content. DJ processed the PET data and revised the manuscript. KC and ER had a major role in statistics supervision and revised the manuscript. $\mathrm{KJ}$ designed the study, acquired the PET data, and revised the manuscript. YTQ designed the study, acquired the data, and revised the manuscript. All authors read and approved the final manuscript.

\section{Ethics approval and consent to participate}

Written informed consents were obtained from all the participants before undergoing any procedure. Approvals were obtained from the University of Antioquia Ethics Committee and the MGH Institutional Review Board before that participants underwent any procedures.

\section{Consent for publication}

Written informed consents were obtained from all the participants for publication of this manuscript. Copies of the written consents are available for review by the Editor-in-Chief of this journal.

\section{Competing interests}

The authors declare that they have no competing interests.

\section{Publisher's Note}

Springer Nature remains neutral with regard to jurisdictional claims in published maps and institutional affiliations.

\section{Author details}

'Massachusetts General Hospital, Harvard Medical School, 149 13th Street, Suite 10.014, Charlestown, Boston, MA 02119, USA. ${ }^{2}$ Cliniques Universitaires Saint-Luc, Université Catholique de Louvain, Brussels, Belgium. ${ }^{3}$ Universidad de Antioquia, Medellín, Colombia. ${ }^{4}$ Brigham and Women's Hospital, Harvard Medical School, Boston, MA, USA. ${ }^{5}$ Banner Alzheimer Institute, Phoenix, AZ, USA.

Received: 6 November 2018 Accepted: 14 January 2019

Published online: 04 February 2019

\section{References}

1. Bateman RJ, et al. Clinical and biomarker changes in dominantly inherited Alzheimer's disease. N Engl J Med. 2012;367:795-804.

2. Fleisher AS, et al. Florbetapir PET analysis of amyloid-beta deposition in the presenilin 1 E280A autosomal dominant Alzheimer's disease kindred: a cross-sectional study. Lancet Neurol. 2012;11:1057-65.

3. Quiroz YT, et al. Association between amyloid and tau accumulation in young adults with autosomal dominant Alzheimer disease. JAMA Neurol. 2018:75(5):548-56

4. Yau WW, et al. Longitudinal assessment of neuroimaging and clinical markers in autosomal dominant Alzheimer's disease: a prospective cohort study. Lancet Neurol. 2015;14:804-13.

5. Klunk WE, et al. Amyloid deposition begins in the striatum of presenilin-1 mutation carriers from two unrelated pedigrees. J Neurosci. 2007;27:6174-84.

6. Villemagne $\mathrm{VL}$, et al. High striatal amyloid beta-peptide deposition across different autosomal Alzheimer disease mutation types. Arch Neurol. 2009;66: 1537-44.

7. Cohen $A D$, et al. Early striatal amyloid deposition distinguishes Down syndrome and autosomal dominant Alzheimer's disease from late-onset amyloid deposition. Alzheimers Dement. 2018;14(6):743-50. 
8. Hanseeuw BJ, et al. PET staging of amyloidosis using striatum. Alzheimers Dement. 2018;14(10):1281-92.

9. Acosta-Baena N, et al. Pre-dementia clinical stages in presenilin 1 E280A familial early-onset Alzheimer's disease: a retrospective cohort study. Lancet Neurol. 2011;10:213-20.

10. Lopera F, et al. Clinical features of early-onset Alzheimer disease in a large kindred with an E280A presenilin-1 mutation. JAMA. 1997;277:793-9.

11. Aguirre-Acevedo DC, et al. Validity and reliability of the CERAD-Col neuropsychological battery. Rev Neurol. 2007;45:655-60.

12. Um YH, et al. A case report of a 37-year-old Alzheimer's disease patient with prominent striatum amyloid retention. Psychiatry Investig. 2017;14:521-4.

13. Knight WD, et al. Carbon-11-Pittsburgh compound B positron emission tomography imaging of amyloid deposition in presenilin 1 mutation carriers. Brain. 2011;134:293-300.

14. Gordon BA, et al. Spatial patterns of neuroimaging biomarker change in individuals from families with autosomal dominant Alzheimer's disease: a longitudinal study. Lancet Neurol. 2018;17:241-50.

Ready to submit your research? Choose BMC and benefit from:

- fast, convenient online submission

- thorough peer review by experienced researchers in your field

- rapid publication on acceptance

- support for research data, including large and complex data types

- gold Open Access which fosters wider collaboration and increased citations

- maximum visibility for your research: over $100 \mathrm{M}$ website views per year

At $\mathrm{BMC}$, research is always in progress.

Learn more biomedcentral.com/submissions 